\title{
Convergence of Two Analytical Flowfield Models Predicting a Destabilizing Agent in Rocket Combustion
}

\author{
J. Majdalani* \\ Marquette University, Milwaukee, Wisconsin 53233 \\ G. A. Flandro \\ University of Tennessee Space Institute, Tullahoma, Tennessee 37388 \\ and \\ T. S. Roh \\ California Institute of Technology, Pasadena, California 91125
}

\begin{abstract}
In the combustion stability assessment of solid propellant rocket motors, several new destabilizing terms are introduced when rotational-flow effects are properly accounted for. Such effects must be included when the wave motion is parallel to the burning surface. A normal fluctuating velocity component then appears in a careful resolution of intrinsic fluid dynamics, including acoustico-vortical interactions that must satisfy mass and momentum conservation principles while accommodating the no-slip condition at the propellant surface. The source of this destabilizing term appears explicitly in two separate, independently derived, analytical formulations of the internal flowfield. Predictions generated by these analytical models are shown to agree with reliable computational data produced recently by a numerical code that solves the unsteady nonlinear Navier-Stokes equations. Verification of the analytical formulations by means of theoretical considerations, numerical comparisons, and global error assessments are also undertaken before examining the impact of the new time-dependent radial-velocity correction on rocket stability. The new radial-velocity fluctuations introduce a correction comparable in importance to the classical pressure coupling at the propellant surface. This effect along with several companion terms must be accounted for properly in the assessment of motor stability characteristics.
\end{abstract}

\begin{tabular}{ll} 
& \multicolumn{1}{c}{ Nomenclature } \\
$A_{p}$ & $=$ dimensional oscillatory pressure amplitude \\
$a_{0}$ & $=$ mean stagnation sound speed, $\sqrt{ }\left(\gamma p_{0} / \rho_{0}\right)$ \\
$\left(\boldsymbol{e}_{r}, \boldsymbol{e}_{z}\right)$ & $=$ radial and axial normal unit vectors \\
$k_{m}$ & $=$ wave number, $m \pi R / L=\omega_{0} R / a_{0}$ \\
$L$ & $=$ internal chamber length \\
$M_{b}$ & $=$ wall injection Mach number, $V_{b} / a_{0}$ \\
$m$ & $=$ pressure oscillation mode number \\
$p$ & $=$ normalized pressure, $p^{*} /\left(\gamma p_{0}\right)$ \\
$p_{0}$ & $=$ mean chamber pressure, $\rho_{0} a_{0}^{2} / \gamma$ \\
$R$ & $=$ dimensional effective radius \\
$R e_{k}$ & $=$ kinetic Reynolds number, $\omega_{0} R^{2} / \nu_{0}$ \\
$r$ & $=$ radial position, $r^{*} / R$ \\
$S r$ & $=$ Strouhal number, $\omega_{0} R / V_{b}=k_{m} / M_{b}$ \\
$t$ & $=$ dimensionless time, $t^{*} a_{0} / R$ \\
$\boldsymbol{U}$ & $=$ Culick's mean flow velocity, ${ }^{6}\left(U_{r}, U_{z}\right)$ \\
$U_{r}$ & $=$ radial mean flow velocity, $-r^{-1} \sin \theta$ \\
$U_{z}$ & $=$ axial mean flow velocity, $\pi z \cos \theta$ \\
$\boldsymbol{u}$ & $=$ total velocity, $\boldsymbol{u}^{*} / a_{0}$ \\
$\hat{\boldsymbol{u}}$ & $=$ dimensionless acoustic velocity, $\hat{\boldsymbol{u}}^{*} / a_{0}$ \\
$\tilde{\boldsymbol{u}}$ & $=$ dimensionless vortical velocity, $\tilde{\boldsymbol{u}}^{*} / a_{0}$ \\
$\boldsymbol{u}^{(1)}$ & $=$ total unsteady velocity, $\boldsymbol{u}^{* 1)} / a_{0}$ is equal to $(\hat{\boldsymbol{u}}+\tilde{\boldsymbol{u}})$ \\
$V_{b}$ & $=$ radial injection speed at the wall \\
$y$ & $=$ distance from the transpiring wall, $1-r$ \\
$z$ & $=$ axial distance from the head end, $z^{*} / R$ \\
$\alpha$ & $=$ order of the global asymptotic error \\
&
\end{tabular}

Presented as Paper 98-3698 at the AIAA 34th Joint Propulsion Conference, Cleveland, OH, 13-15 July 1998; received 16 July 1998; revision received 1 April 1999; accepted for publication 1 June 1999. Copyright @ 1999 by the authors. Published by the American Institute of Aeronautics and Astronautics, Inc., with permission.

${ }^{*}$ Assistant Professor, Department of Mechanical and Industrial Engineering. Member AIAA.

Boling Chair Professor of Mechanical and Aerospace Engineering. Associate Fellow AIAA.

${ }^{\ddagger}$ Post-Doctoral Scholar, Mechanical Engineering and Jet Propulsion Center. Member AIAA.

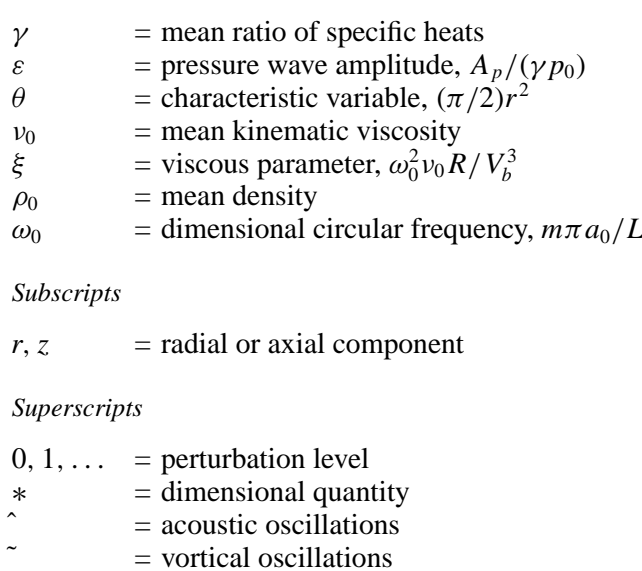

\section{Introduction}

$\mathrm{N}$ combustion stability assessments of solid rocket motors, the source of radial-velocity fluctuations is frequently attributed to pressure oscillations at the propellant surface that, through the pressure-coupling mechanism, namely, $\dot{m} \sim p^{n}$, can induce an oscillatory component in the radial direction. This is done sometimes without giving due consideration to certain fluid dynamical interactions within the motor. In particular, effects related to the production of unsteady vorticity are lost in the assumption that the unsteady flow is a perturbed acoustic wave and, hence, irrotational. This drawback is removed in two multidimensional formulations of the time-dependent field that correctly satisfy applicable conservation laws. As derived independently by Flandro ${ }^{1}$ using regular perturbations and Majdalani and Van Moorhem ${ }^{2}$ using multiplescale expansions, two explicit formulations for the time-dependent field are now available that satisfy the appropriate conservation principles. In addition, these expressions agree very well with computational predictions obtained from full, compressible, nonlinearized Navier-Stokes solvers. ${ }^{3,4}$ 
An important consequence of introducing the unsteady rotationalflow corrections is the appearance of new terms in the stability assessment of the chamber. ${ }^{1}$ One of these is a radial-velocity fluctuation with an amplitude proportional to the mean-flow Mach number. Hence, it has the same importance in the system stability as the pressure-coupling effect (which exhibits a similar influence). In this paper we demonstrate that this term arises in two separate formulations of the problem. A careful numerical verification is also undertaken in the hope of resolving possible doubts regarding its physical origin. This paper begins by reviewing briefly in Sec. I the two distinct analytical formulations of the unsteady rotational flow in a rocket chamber. Because the correctness of either model depends largely on how accurately it can replicate reality, a careful verification process is undertaken in Sec. III. This verification is threefold and includes theoretical, computational, and global error validations. The theoretical verification consists of ensuring that flowfield components satisfy continuity. Computational validations involve direct comparisons with recently acquired computational fluid dynamics (CFD) data from a nonlinear Navier-Stokes solver. These comparisons illustrate the reassuring agreement between analytical and computational results. The global or total error that accompanies each individual formulation is carefully determined in addition to the order associated with each method. Having established with certainty the reported accuracy, Sec. IV focuses on a key flowfield component that produces a destabilizing effect that is of the same order of magnitude as that attributed to pressure coupling. The characteristics of this radial-velocity fluctuation are described, emphasizing the nonzero value it assumes at the propellant surface, that is, the edge of the combustion zone. The direct impact on stability is covered in Sec V.

\section{Analytical Models}

Following Flandro's nomenclature, ${ }^{5}$ the total velocity is expressed in two-dimensional axisymmetric coordinates after normalizing by the speed of sound

$$
\boldsymbol{u}(r, z, t)=M_{b} \boldsymbol{U}(r, z)+\varepsilon \boldsymbol{u}^{(1)}(r, z, t)
$$

where $\boldsymbol{U}(r, z)$ is Culick's mean-flow profile, ${ }^{6} M_{b}$ is the injection Mach number at the wall, and $\varepsilon=A_{p} /\left(\gamma p_{0}\right)$ is the normalized pressure-wave amplitude representing the primary perturbation parameter used to linearize the Navier-Stokes equations. From the axial and normal components of the velocity, the total unsteady component is

$$
\boldsymbol{u}^{(1)}(r, z, t)=u_{r}^{(1)} \boldsymbol{e}_{r}+u_{z}^{(1)} \boldsymbol{e}_{z}
$$

\section{A. Regular-Perturbation (RP) Formulation}

Inasmuch as derivation details are presented in separate papers, ${ }^{1,5}$ the goal now is to focus on the results. The time-dependent expressions for the velocity field are repeated here for the reader's convenience, as originally published,

$$
\begin{aligned}
& u_{z}^{(1)}(r, z, t)=\sin \left(k_{m} z\right) \sin \left(k_{m} t\right) \\
& \quad+\left(B^{r} \sin \varphi_{0}-B^{i} \cos \varphi_{0}\right) r \exp \left(\phi^{r}\right) \sin \left(k_{m} z \sin \theta\right) \\
& u_{r}^{(1)}(r, z, t)=M_{b} r^{2} U_{r}^{2}\left(B^{r} \cos \varphi_{0}+B^{i} \sin \varphi_{0}\right) \\
& \quad \times \exp \left(\phi^{r}\right) \cos \left(k_{m} z \sin \theta\right)
\end{aligned}
$$

where

$$
\begin{gathered}
\xi=\frac{\omega_{0}^{2} R v_{0}}{V_{b}^{3}}, \quad \theta=\frac{\pi}{2} r^{2}, \quad U_{r}=-r^{-1} \sin \theta \\
\varphi_{0}=k_{m} t+(5 r / \pi) \ln \tan (\theta / 2) \\
\phi^{r}=\left(-\xi / \pi^{2}\right)\left[\left(2 \pi^{2} / S r^{2}-1\right) I(\theta)+\csc \theta+(\pi / 2)\left(\cos \theta / U_{r}^{2}\right)\right]
\end{gathered}
$$

$$
\begin{gathered}
I(\theta) \cong \theta+\frac{1}{18} \theta^{3}+\frac{7}{1800} \theta^{5}+\frac{31}{105840} \theta^{7}+\cdots \\
C^{r}=\frac{\left(S r^{2}+\xi\right) S^{3} \exp \left[-\phi^{r}(1)\right]}{\left(S r^{2}+\xi\right)^{2}+\xi^{2} S r^{2}} \\
C^{i}=\frac{\xi S^{4} \exp \left[-\phi^{r}(1)\right]}{\left(S r^{2}+\xi\right)^{2}+\xi^{2} S r^{2}} \\
B^{r}=C^{r} \frac{U_{r}}{S r}+C^{i} \frac{\xi}{S r^{2} U_{r}}, \quad B^{i}=C^{i} \frac{U_{r}}{S r}-C^{r} \frac{\xi}{S r^{2} U_{r}} \\
\phi^{r}(1)=\xi\left(0.0842922527-3.66386237665 / S r^{2}\right)
\end{gathered}
$$

These results are accurate to $\mathcal{O}(1 / \mathrm{Sr})$, the reciprocal of the Strouhal number $\left(\operatorname{Sr}=\omega_{0} R / V_{b}\right)$, which is always smaller than about $10 \%$ by virtue of $S r>10$. The global error, thus, decreases at higher oscillation frequencies, larger effective radii, and smaller injection speeds. It approaches the exact solution at higher oscillation modes, which are very difficult to resolve numerically.

A shorter version of the regular perturbation (RP) solution can be formulated at the expense of a loss in accuracy that is insignificant at high kinetic Reynolds numbers. The outcome consisting of

$u_{z}^{(1)}(r, z, t)=\sin \left(k_{m} z\right) \sin \left(k_{m} t\right)+r U_{r} \exp \left(\phi^{r}\right) \sin \varphi_{o} \sin \left(k_{m} z \sin \theta\right)$

$$
u_{r}^{(1)}(r, z, t)=M_{b} r^{2} U_{r}^{3} \exp \left(\phi^{r}\right) \cos \varphi_{o} \cos \left(k_{m} z \sin \theta\right)
$$

carries a maximum error bound of $18 \%$ for $R e_{k}=5 \times 10^{5}$ and $S r=100$. The order of the error associated with Eqs. (13) and (14) approaches $1 / S r$ asymptotically as $R_{k} \rightarrow \infty$.

\section{B. Multiple-Scale (MS) Formulation}

Based on a two-variable derivative expansion procedure, an alternative solution is furnished by Majdalani and Van Moorhem ${ }^{2}$

$$
u_{z}^{(1)}(r, z, t)=\sin \left(k_{m} z\right) \sin \left(k_{m} t\right)+r U_{r} \sin \left(k_{m} z \sin \theta\right) \exp (\zeta) \sin \varphi_{0}
$$

$$
u_{r}^{(1)}(r, z, t)=M_{b} r^{2} U_{r}^{3} \cos \left(k_{m} z \sin \theta\right) \cos \varphi_{0} \exp (\zeta)
$$

where the spatial damping term $\zeta=\xi \eta U_{r}^{-3}$ is based on an effective scale function that controls the solution,

$$
\eta=y\left[1+c y^{c}\left(y r^{-1}-c \ln r\right)\right]^{-1}, \quad y=1-r, \quad c=\frac{3}{2}
$$

Note that the global error associated with Eqs. (15) and (16) is $\mathcal{O}\left(1 / R e_{k}\right) \equiv \mathcal{O}\left(v_{0} \omega_{0}^{-1} R^{-2}\right)$. The latter is a very small quantity, independent of the injection speed, being the square of the ratio of the thin hard wall Stokes layer thickness $\left[\sqrt{ }\left(v_{0} / \omega_{0}\right)\right]$ and the chamber radius. The error, thus, decreases at higher oscillation modes and larger radii. In comparison to the Strouhal number, the kinetic Reynolds number is a much larger quantity because the ratio

$$
\frac{R e_{k}}{S r}=\frac{\omega_{0} R^{2} / v_{0}}{\omega_{0} R / V_{b}}=\frac{V_{b} R}{v_{0}} \gg 1
$$

results in the classic injection Reynolds number, which is always significant. From a practical standpoint, the global errors associated with both models remain too small to affect the overall assessment by an appreciable amount. This will be confirmed in the forthcoming assessments.

\section{Verifications}

To confirm the accuracy reported in the preceding section, we undertake analytical and computational verifications along with a careful error analysis to ascertain the order of the global error associated with each analytical formulation. 


\section{A. Analytical Verification}

From mass conservation, both steady and time-dependent components of the total velocity must satisfy continuity. The steady function $\boldsymbol{U}(r, z)$ is clearly solenoidal, that is, divergence free, and satisfies $\nabla \cdot \boldsymbol{U}=0$. Satisfaction of mass conservation by the timedependent field requires a more delicate assessment. In fact, one must recall ${ }^{5,7-9}$ that the temporal component $\boldsymbol{u}^{(1)}(r, z, t)$ is the sum of two fluctuations: an irrotational, pressure-driven part $\hat{\boldsymbol{u}}$ and a rotational, vorticity-driven part $\tilde{\boldsymbol{u}}$

On one hand, the pressure-driven part corresponding to either asymptotic formulations is $\hat{\boldsymbol{u}}=\sin \left(k_{m} z\right) \sin \left(k_{m} t\right) \boldsymbol{e}_{z}$. This must satisfy the conservation of mass written, at $\mathcal{O}\left(M_{b}\right)$, for the acoustic field $^{2}$

$$
\frac{\partial \hat{\boldsymbol{u}}}{\partial t}+\nabla \hat{p}=\mathcal{O}\left(M_{b}\right)
$$

The preceding relationship does not pose a problem inasmuch as one may verify, by mere inspection, that Eq. (19) is true for acoustic pressure oscillations of the type assumed here, namely, when $\hat{p}=\cos \left(k_{m} z\right) \cos \left(k_{m} t\right)$.

On the other hand, the vorticity-driven part is given by $\tilde{\boldsymbol{u}}=M_{b} r^{2} U_{r}^{2}\left(B^{r} \cos \varphi_{0}+B^{i} \sin \varphi_{0}\right) \exp \left(\phi^{r}\right) \cos \left(k_{m} z \sin \theta\right) \boldsymbol{e}_{r}$

$$
\left.+\left(B^{r} \sin \varphi_{0}-B^{i} \cos \varphi_{0}\right) r \exp \left(\phi^{r}\right) \sin \left(k_{m} z \sin \theta\right)\right) \boldsymbol{e}_{z}
$$

following the RP approach and by

$$
\tilde{\boldsymbol{u}}=M_{b} r^{2} U_{r}^{3} \cos \left(k_{m} z \sin \theta\right) \cos \varphi_{0} \exp (\zeta) \boldsymbol{e}_{r}
$$

$$
+r U_{r} \sin \left(k_{m} z \sin \theta\right) \exp (\zeta) \sin \varphi_{0} \boldsymbol{e}_{z}
$$

following the multiple-scale (MS) approach. Evidently, both Eqs. (20) and (21) must satisfy independently the conservation of mass written, at $\mathcal{O}\left(M_{b}\right)$, for the rotational field ${ }^{2}$

$$
\nabla \cdot \tilde{\boldsymbol{u}}=\frac{\partial \tilde{u}_{r}}{\partial r}+\frac{\tilde{u}_{r}}{r}+\frac{\partial \tilde{u}_{z}}{\partial z}=0
$$

Here also, one can show, without much difficulty, that both Eqs. (20) and (21) satisfy, in their leading-order terms, the continuity equation expressed by Eq. (22)

\section{B. Computational (CFD) Verification}

In previous work, ${ }^{2,3}$ the analytical expressions just reported were found to concur, as they should, with the numerical solution of their governing, linearized Navier-Stokes equations. A recen comparison with computational data acquired from a full, nonlinearized Navier-Stokes solver developed by Roh et al. ${ }^{10}$ and Roh and Culick ${ }^{11}$ shows very good agreement between analytical and computational predictions.

Comparisons are shown in Fig. 1 for a typical case at the firs two oscillation modes. The physical parameters correspond to an average tactical rocket motor proposed by Flandro. ${ }^{1}$ Additional test parameters include a chamber pressure of $5.066 \times 10^{6} \mathrm{~N} \mathrm{~m}^{-2}$ (50 atm), a density of $6.586 \mathrm{~kg} \mathrm{~m}^{-3}$, a kinematic viscosity of $7.69 \times 10^{-6} \mathrm{~m}^{2} \mathrm{~s}^{-1}$, a temperature of $2000 \mathrm{~K}$, a ratio of specific heats of 1.3 , a Prandtl number of 0.9 , a speed of sound of $1000 \mathrm{~m} \mathrm{~s}^{-1}$, and an injection speed of $3 \mathrm{~m} \mathrm{~s}^{-1}$. The CFD scheme employs a grid system that includes $60 \times 150$ nodes (in the axial and radial directions, respectively) for mode 1 and $80 \times 240$ nodes for mode 2 , with clustering ratio that increases with distance from the wall. This is necessitated by the need to resolve rapid variations that occur away from the wall as the spatial wavelength of vortical waves diminishes, being a direct function of Culick's radial velocity. ${ }^{3}$ Because the spatial wavelength gradually vanishes as the centerline is approached, an increasingly larger number of nodes will be needed to maintain a uniform numerical error. A highly refined grid quickly becomes unaffordable due to increased memory requirements and CPU time.
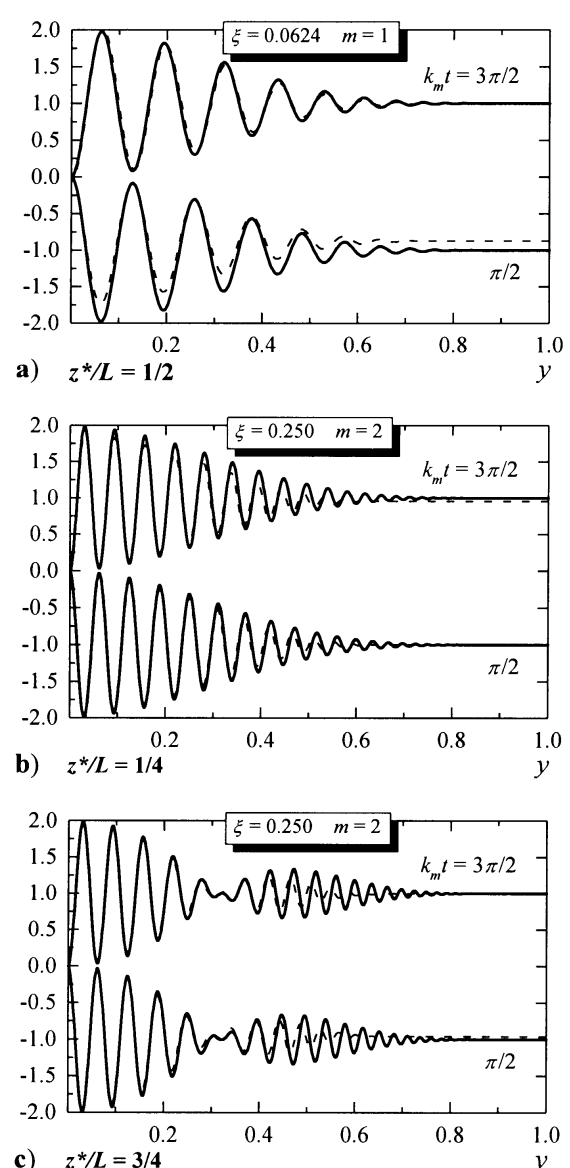

Fig. 1 Unsteady axial velocity obtained analytically ( - ) and from Navier-Stokes solvers (---) overlaid at two evenly spaced times in a typical tactical rocket motor. Results are for the first two oscillation modes evaluated at the first and last acoustic pressure nodes.

In Fig. 1, the expected buildup in numerical error as the centerline is approached is attributed to the rough grid resolution used to generate the CFD data. This slight discrepancy, owing to unavoidable numerical errors, does not undermine the surprisingly good agreement with analytical predictions.

Another contribution to such deviations can be attributed to the finite time discretization, used in the numerical scheme, which allows producing CFD data at approximate times. Whereas analytical predictions are shown at two instants separated by a phase difference that is exactly equal to $\pi$, numerical predictions are furnished at approximate phase differences.

Finally, one must not forget that the analytical models are solutions to the linearized Navier-Stokes equations. As such, they cannot account for the small nonlinearity effects that are captured by numerical simulations. Overall, within computational uncertainty, the data indicate that, when turbulence is ignored, the analytical models tend to represent fairly well the nature of the internal flowfield. Representative data sets used to generate one time evolution in Fig. 1a are listed in Table 1 for confirmatory purposes.

\section{Absolute Error Verification}

In a recent paper, Bosely ${ }^{12}$ describes a rigorous technique that can be employed to verify the error associated with an asymptotic solution. His technique serves two purposes: 1) to evaluate the order of the error and 2) to ensure that the final expression is free from human errors. His technique is used here to verify the error associated with both RP and MS formulations, in the hope of dispelling 
Table 1 Unsteady axial velocity predictions

\begin{tabular}{lccc}
\hline \hline$y$ & RP & MS & CFD \\
\hline 1.0 & 1.00000 & 1.00000 & 1.00000 \\
0.9 & 1.00020 & 1.00020 & 1.00073 \\
0.8 & 0.99560 & 0.99584 & 1.00069 \\
0.7 & 0.98662 & 0.98858 & 0.99667 \\
0.6 & 1.04700 & 1.04160 & 1.02463 \\
0.5 & 0.89730 & 0.88565 & 0.90050 \\
0.4 & 0.86056 & 0.83989 & 0.82500 \\
0.3 & 1.30870 & 1.28400 & 1.19759 \\
0.2 & 1.77650 & 1.78480 & 1.79982 \\
0.1 & 0.80323 & 0.82064 & 0.83152 \\
0.0 & $5.00 E-5$ & $6.3 E-12$ & 0.00688 \\
\hline
\end{tabular}

Table 2 Maximum error for a tactical motor

\begin{tabular}{lcc}
\hline \hline$S r=\omega_{0} R / V_{b}$ & $\mathrm{RP}$ & $\mathrm{MS}$ \\
\hline 10.000000 & 0.1285786 & $3.39398 E-5$ \\
12.589258 & 0.0946560 & $5.61192 E-5$ \\
15.848941 & 0.0876632 & $8.44848 E-5$ \\
19.952633 & 0.0723662 & $1.31394 E-4$ \\
25.118875 & 0.0547265 & $2.22025 E-4$ \\
31.622753 & 0.0458222 & $3.54939 E-4$ \\
39.810661 & 0.0359189 & $5.99515 E-4$ \\
50.118782 & 0.0290877 & 0.0010290 \\
63.095862 & 0.0236003 & 0.0017954 \\
79.432534 & 0.0195297 & 0.0032031 \\
100.00000 & 0.0167755 & 0.0057601 \\
\hline \hline
\end{tabular}

remaining doubts regarding the declared accuracy reported in the preceding paragraphs.

The way this technique works is quite straightforward. It starts by calculating the maximum absolute error, defined as the absolute difference between analytical and numerical solutions,

$$
E_{\max }=\max _{0 \leq r \leq 1}\left|u_{\text {numeric }}^{(1)}-u_{\text {analytic }}^{(1)}\right|
$$

where $u_{\text {numeric }}^{(1)}$ is the solution to the linearized momentum equation over the normalized interval $0 \leq r \leq 1$. As a result of using a seventhorder Runge-Kutta technique and a mesh size of $10^{-6}$, the error associated with calculating $u_{\text {numeric }}^{(1)}$ is negligible. This enables us to use $u_{\text {(1) }}$ as a benchmark against which asymptotic solutions can be compared. Thus, if $E_{\max }$ is of $\mathcal{O}(1 / S r)$, as foretold by the RP solution, one can write

$$
E_{\max }=K(1 / S r)^{\alpha}
$$

and show that $\alpha \rightarrow 1$ for large $S r$. The order of the error can be determined from the slope of $E_{\max }$ vs $1 / S r$ plotted on a log-log scale. This slope can be determined quite accurately by using, for instance, the method of least squares.

If, on the other hand, $E_{\max }$ is of $\mathcal{O}\left(1 / R e_{k}\right)$, as predicted theoretically for the MS formulation, then one could set

$$
E_{\max }=K\left(1 / R e_{k}\right)^{\alpha}
$$

and show that $\alpha \rightarrow 1$ for large $R e_{k}$.

The maximum error is, thus, evaluated and shown in Fig. 2 for the RP (Fig. 2a) and MS (Fig. 2b) formulations. For the RP case, the error decreases and its order approaches unity with large Strouha and kinetic Reynolds numbers. It deteriorates for small Strouha numbers. For the MS formulation, the same occurs for decreasing Strouhal and increasing kinetic Reynolds numbers. In a sense, the errors from both models are complementary with respect to the Strouhal number. This is clearly seen in Fig. 3, where errors from both models are shown at discrete values of the Strouhal number. In the same vein, a typical comparison of maximum absolute errors obtained for a tactical rocket motor with $R e_{k}=2.1 \times 10^{6}$ is furnished in Table 2.

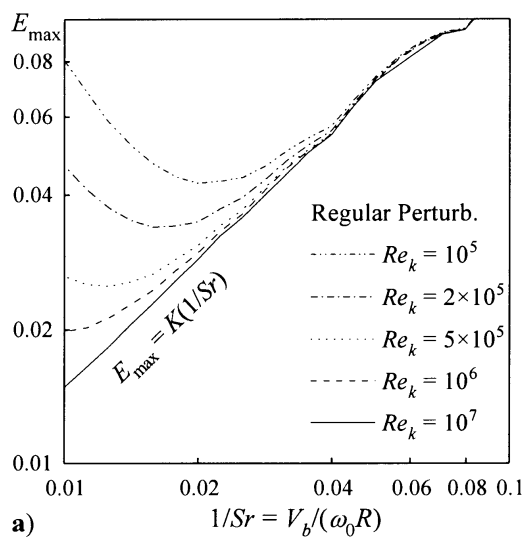

a)

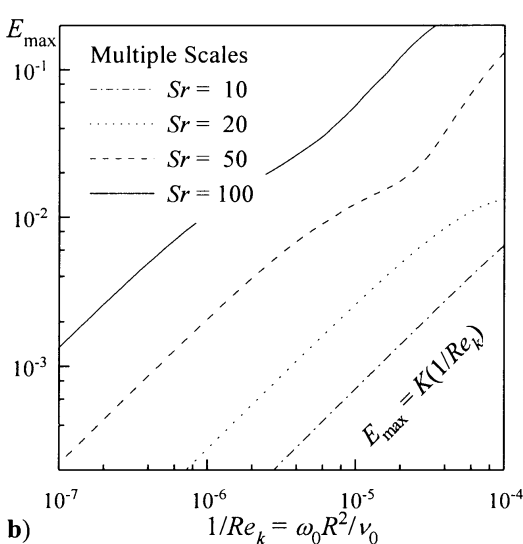

Fig. 2 Maximum absolute error associated with a) Flandro's ${ }^{1}$ RP and b) Majdalani and Van Moorhem's ${ }^{2}$ MS formulations.

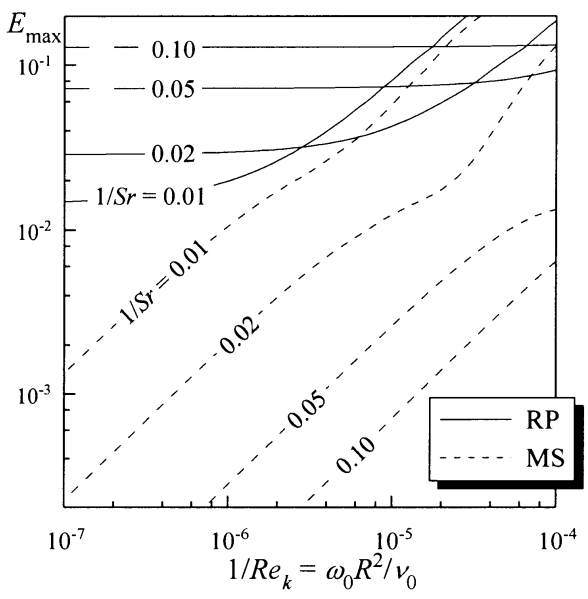

3 Comparison of maximum absolute errors associated with Flandro' ${ }^{1}$ and Majdalani and Van Moorhem's ${ }^{2}$ formulations.

\section{Nonzero Radial Fluctuations}

In this section, we address the character of unsteady radial velocities obtained from both analytical models. Before addressing the influence of this velocity component on stability, we carry out a comparative verification process to confirm the accuracy associated with both explicit formulations.

The explicit radial velocities expressed by Eqs. (4) and (16), derived totally separately, are compared to each other and to the corresponding numerical solution, for three cases reported in Ref. 1 that span a wide range of rocket motors. These comparisons are drawn in Fig. 4 for the first oscillation mode at $z^{*} / L=15 \%$, where the flowfield is predominantly laminar. 

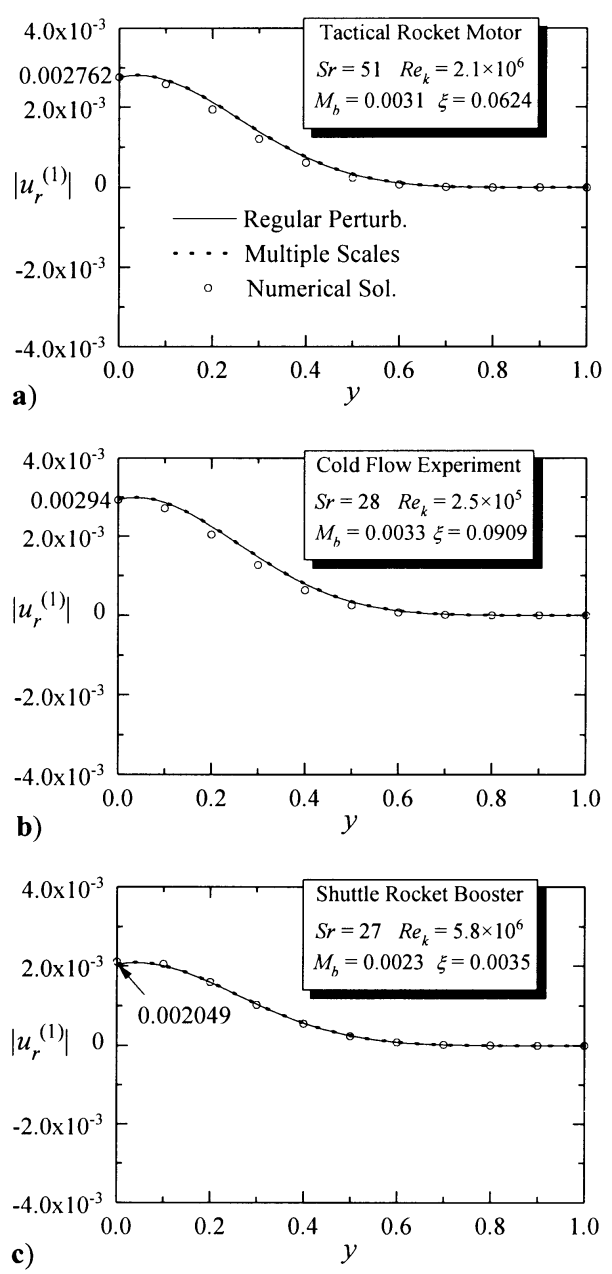

Fig. 4 Modulus of radial velocity fluctuations from both RP and MS formulations compared with numerical predictions; comparisons correspond to $m=1$ and $z^{*} / L=0.15$

Clearly, both RP and MS formulations coincide everywhere with the numerical solution. The same level of agreement persists a higher oscillation modes and at different locations within the chamber (not shown here). The modulus of the rotational radial velocity expressed in complex notation, which is plotted in Fig. 4, is largest at the wall and decays as the centerline is approached. This is to be expected because the vorticity-coupled axial counterpart decays and vanishes when approaching the centerline as well. In particular, note that the nonzero value of the radial velocity at the wall negates traditional one-dimensional flowfield predictions that attribute velocity fluctuations to the pressure-coupling response. This value for $u_{r}^{(1)}$ can be determined from either Eq. (4) or Eq. (16). The result is

$$
u_{r}^{(1)}(1, z, t)=-M_{b} \cos \left(k_{m} z\right)=-M_{b} \cos \left(m \pi z^{*} / L\right)
$$

which, for $z^{*} / L=15 \%$, is $-0.891 M_{b}$. In Fig. 4 , this value of the radial-velocity amplitude at the wall is indicated for further emphasis. The graphical agreement shown in Fig. 4 is presented in tabular format for one case in Table 3, where data corresponding to the Shuttle rocket booster (SRB) are listed at 10 evenly spaced points. Note that the agreement with computational data is very good near the wall and deteriorates as we approach the centerline due to progressive buildup in the numerical error for reasons already stated in Sec. III.B. These solutions concur in both amplitude and phase, as shown in Fig. 5, where radial velocity profiles are compared a three evenly spaced times for a typical tactical rocket motor. The agreement between RP and MS predictions is remarkable and in-
Table 3 Unsteady radial velocity amplitudes (SRB)

\begin{tabular}{llll}
\hline \hline$y$ & \multicolumn{1}{c}{ RP } & \multicolumn{1}{c}{ MS } & Numerical \\
\hline 1.0 & 0.00000000 & 0.00000000 & 0.00000000 \\
0.9 & $8.53137 E-8$ & $8.52946 E-8$ & $5.63200 E-8$ \\
0.8 & $2.81421 E-6$ & $2.81590 E-6$ & $2.46140 E-6$ \\
0.7 & $2.12909 E-5$ & $2.12990 E-5$ & $1.95797 E-5$ \\
0.6 & $8.75767 E-5$ & $8.75969 E-5$ & $8.21043 E-5$ \\
0.5 & $2.53138 E-4$ & $2.53175 E-4$ & $2.40537 E-4$ \\
0.4 & $5.70289 E-4$ & $5.70344 E-4$ & $5.49830 E-4$ \\
0.3 & 0.00104740 & 0.00104750 & 0.00102800 \\
0.2 & 0.00159440 & 0.00159450 & 0.00159710 \\
0.1 & 0.00200820 & 0.00200830 & 0.00204940 \\
0.0 & 0.00204930 & 0.00204930 & 0.00210990 \\
\hline \hline
\end{tabular}

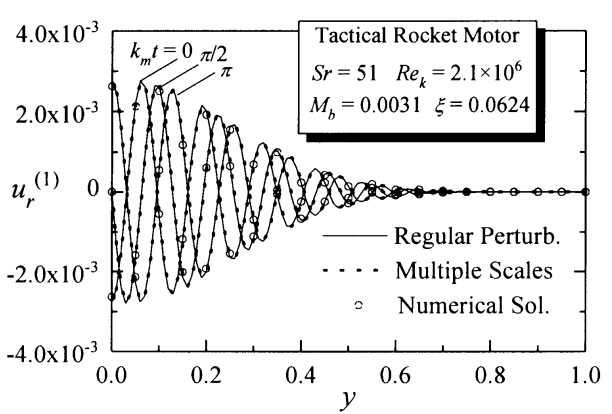

Fig. 5 Radial velocity profiles at three different phases separated by 90 deg. Results are obtained from RP, MS, and numerical predictions; comparisons correspond to a typical tactical rocket motor with $m=1$ and $z^{*} / L=\mathbf{0 . 1 5}$.

cludes, in some cases, matching digits up to the eighth decimal place.

The counterintuitive appearance of a nonzero radial componen at the wall is decreed by the mass conservation requirement in the time-dependent fluctuations. In retrospect, examination of Eq. (22) reveals that

$$
\frac{\partial \tilde{u}_{r}}{\partial r}+\frac{\tilde{u}_{r}}{r}=-\frac{\partial \tilde{u}_{z}}{\partial z}
$$

which indicates that the existence of a nonzero $\tilde{u}_{r}$ can stem from a nonzero axial variation in $\tilde{u}_{z}$ at the wall. Because the axial vortical component $\tilde{u}_{z}$ varies along the wall in the streamwise direction $z$, the transverse component $\tilde{u}_{r}$ is forced, via continuity, to assume whatever finite value is necessary to counterbalance the axial gradient.

\section{Stability Implications}

Three different methods have been employed to determine the part of the radial-velocity fluctuation that is created by rotational-flow corrections. In assessing the motor system stability characteristics, this radial-velocity component must be added to others that arise in the irrotational (acoustic) part of the problem. In particular, terms that appear as a result of pressure-sensitive combustion effects represent separate elements of the radial-velocity fluctuation.

It should be apparent that the three separate calculations of the radial rotational velocity fluctuation are in substantial agreement What is most important is that the predicted value of the radial velocity at the surface, $-M_{b} \cos \left(m \pi z^{*} / L\right)$, is proportional to the mean-flow Mach number as already described. That is, it is of the same order of magnitude as other key ingredients of the system gain or loss balance. The velocity fluctuation is in phase with the pressure fluctuation. It therefore, has the same significance as pressure coupling in the stability of the motor because the response function is also proportional to the mean-flow Mach number.

The new destabilizing term was discussed in detail in Ref. 5 , where its inclusion was demonstrated to improve the agreement between stability predictions and experimental data for motor systems that exhibit linear stability behavior. 


\section{Conclusion}

What has been accomplished here is the verification that a new destabilizing agent must be included in linear stability calculations for cases with wave motion parallel to the burning surfaces. The new findings are not the result of double bookkeeping as some critics contend. They represent natural gas motions that must be present when the no-slip boundary conditions at the surfaces are accommodated. They arise in the same set of interactions that lead to the much discussed flow turning damping effect.

\section{Acknowledgments}

The authors wish to thank Fred E. C. Culick from the California Institute of Technology and Vigor Yang from the Pennsylvania State University for their helpful collaboration, constructive comments, and generous production of Navier-Stokes data that made comparisons to nonlinearized computational fluid dynamics predictions possible.

\section{References}

${ }^{1}$ Flandro, G. A., “On Flow Turning,” AIAA Paper 95-2530, July 1995. ${ }^{2}$ Majdalani, J., and Van Moorhem, W. K., "Improved Time-Dependent Flowfield Solution for Solid Rocket Motors," AIAA Journal, Vol. 36, No. 2 , 1998 , pp. 241-248

${ }^{3}$ Majdalani, J., "Characterization of the Laminar Boundary Layer in Solid Rocket Motors," AIAA Paper 98-3699, July 1998.
${ }^{4}$ Majdalani, J., "The Boundary Layer Structure in Cylindrical Rocket Motors," AIAA Journal, Vol. 37, No. 4, 1999, pp. 505-508.

5Flandro, G. A., "Effects of Vorticity on Rocket Combustion Stability," Journal of Propulsion and Power, Vol. 11, No. 4, 1995, pp. 607-625.

${ }^{6}$ Culick, F. E. C., "Rotational Axisymmetric Mean Flow and Damping of Acoustic Waves in a Solid Propellant Rocket," AIAA Journal, Vol. 4, No. 8, 1966, pp. $1462-1464$ ${ }^{7}$ Chu, B. T., and Kovásznay, L. S. G., "Nonlinear Interactions in a Viscous
Heat-Conducting Compressible Gas," Journal of Fluid Mechanics, Vol. 3, 1957, pp. 494-514.

${ }^{8}$ Carrier, B. T., and Carlson, F. D., "On the Propagation of Small Disturbances in a Moving Compressible Fluid," Quarterly of Applied Mathematics, Vol. 4, No. 1, 1946, pp. 1-12.

'Flandro, G. A., "Effects of Vorticity Transport on Axial Acoustic Waves in a Solid Propellant Rocket Chamber," Combustion Instabilities Driven by Thermo-Chemical Acoustic Sources, edited by A. S. Hersh, I. Catton, and R. F. Keltie, Noise Control and Acoustics, Vol. 4, American Society of Mechanical Engineers, New York, 1989, pp. 53-61.

${ }^{10}$ Roh, T. S., Tseng, I. S., and Yang, V., "Effects of Acoustic Oscillations on Flame Dynamics of Homogeneous Propellants in Rocket Molations on Flame Dynamics of Homogeneous Propellants in Rocket Mo-
tors," Journal of Propulsion and Power, Vol. 11, No. 4, 1995, pp. 640650.

${ }^{11}$ Roh, T. S., and Culick, F. E. C., "Transient Combustion Response of Homogeneous Propellants to Acoustic Oscillations in Axisymmetric Rocket Motors," AIAA Paper 97-3325, July 1995.

${ }^{12}$ Bosley, D. L., "A Technique for the Numerical Verification of Asymptotic Expansions," SIAM Review, Vol. 38, No. 1, 1996, pp. 128-135. 